\title{
Multi-mode horn design and beam characteristics for the Planck satellite
}
J.A. Murphy, ${ }^{a, 1}$ T. Peacocke, ${ }^{a}$ B. Maffei, ${ }^{b}$ I. McAuley, ${ }^{a}$ F. Noviello,,${ }^{a, c}$ V. Yurchenko,,${ }^{a, j}$
P.A.R. Ade,${ }^{d}$ G. Savini, ${ }^{d, i}$ J-M. Lamarre,${ }^{e}$ J. Brossard, ${ }^{f, g}{ }^{g}$ R. Colgan, ${ }^{a}$ E. Gleeson, ${ }^{a}$ A.E. Lange, ${ }^{h}$ Y. Longval, ${ }^{c}$ G. Pisano, ${ }^{b}$ J-L. Puget, ${ }^{c}$ I. Ristorcelli, ${ }^{f}$ R. Sudiwala ${ }^{d, h}$ and R.J. Wylde ${ }^{k}$

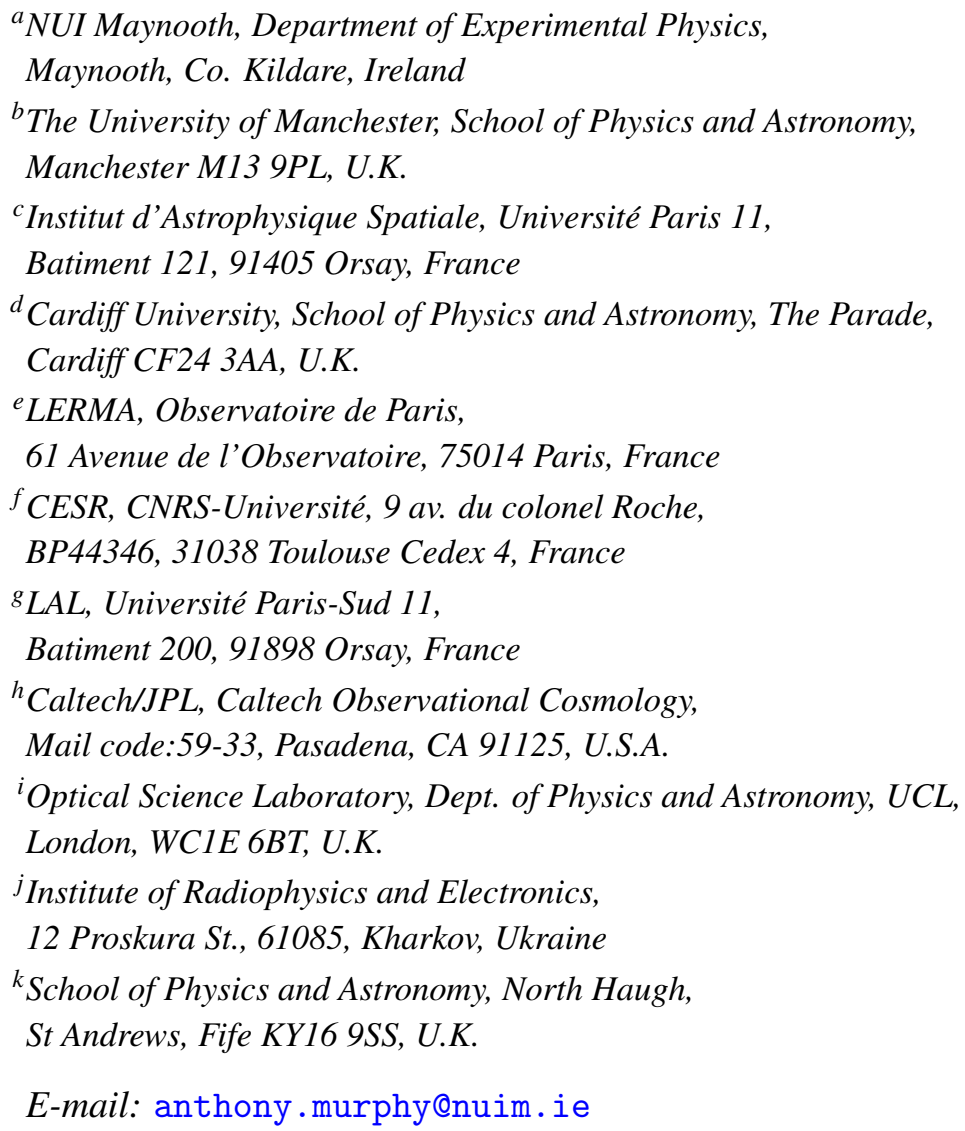

ABSTRACT: The ESA Planck satellite has begun studying the anisotropies of the cosmic microwave background radiation over the whole sky with unprecedented sensitivity and high angular resolution. The High Frequency Instrument, HFI, on Planck is observing simultaneously in six bands

\footnotetext{
${ }^{1}$ Corresponding author.
} 
in the range $100 \mathrm{GHz}$ to $857 \mathrm{GHz}$. The inclusion of non-CMB bands allows for robust removal of foreground sources from the data. This paper is concerned with the design, modeling and predicted performances of the two highest frequency channels centered on $545 \mathrm{GHz}$ and $857 \mathrm{GHz}$, which use specialized multi-mode feedhorns, and are dedicated to observing these foregrounds. Multi-mode systems have the advantage of increasing the throughput, and thus sensitivity, of the detection assembly when diffraction limited resolution is not required. The horns are configured in a back-to-back setup which transmits the signal through filters to a detector horn. The modeling of the broadband beam patterns on the sky is shown to require careful analysis. Simulations of the complex interactions of the horns is computationally challenging when the detector horn in the relay system is included. The paper describes the approach to modeling these high frequency channels and discusses how the optical requirements on the horn designs are met in terms of spillover, edge taper, illumination of the telescope aperture and beam patterns on the sky.

KEYWORDS: Microwave Antennas; Space instrumentation; Optics; Waveguides 


\section{Contents}

1 Introduction 1

2 Optical considerations and design constraints 3

3 Modelling of multi-mode horns 5

4 Telescope illumination patterns and laboratory tests 9

5 Telescope main beam patterns 14

6 Discussion and conclusions $\quad 19$

\section{Introduction}

The primary objective of the Planck mission ${ }^{1}$ is to study the Cosmic Microwave Background (CMB) with the unprecedented sensitivity possible from deep space [1, 2]. The extended frequency coverage of the two instruments on board Planck (the Low Frequency Instrument or LFI [3] and High Frequency Instrument or HFI, [4]) will also improve our understanding of far-infrared foregrounds, both extragalactic sources and the gas and dust of our own galaxy. This is important for achieving the ultimate sensitivity because Planck's measurement noise on the CMB is lower than the level of contamination expected from the foregrounds, even in clean regions of the sky.

This paper is specifically concerned with the design and predicted performances of the two highest frequency channels of the HFI, which use specialized multi-mode feedhorns [5-8]. These channels are dedicated to observing the foreground sources referred to above. For these two high frequency multi-mode bands centred on $545 \mathrm{GHz}$ and $857 \mathrm{GHz}$ (each with a $30 \%$ bandwidth) the telescope is the main source of thermal radiation and photon noise, and the design of these horns requires a very special approach.

The HFI bolometers [9] are incoherent detectors and thus absorb the total power in the propagating modes transmitted by the feed systems which optically couple the detectors through corrugated waveguide structures to an off-axis Gregorian telescope [1], see figure 1. The telescope primary mirror has a projected diameter of $1.5 \mathrm{~m}$ which could provide a diffraction limited resolution of the order 2-3 arcmin for the two bands at $545 \mathrm{GHz}$ and $857 \mathrm{GHz}$. However, there is a requirement to maintain a beam width close to 5 arcmin to minimize the number of positional changes of the satellite and to keep the data rate within the available downlink specification. That allows a multi-mode detection scheme to be implemented, making use of partially coherent optics [10]

\footnotetext{
${ }^{1}$ Planck (http://www.esa.int/Planck) is an ESA project with instruments provided by two scientific consortia funded by ESA member states (in particular the lead countries: France and Italy) with contributions from NASA (USA), and telescope reflectors provided in a collaboration between ESA and a scientific consortium led and funded by Denmark.
} 


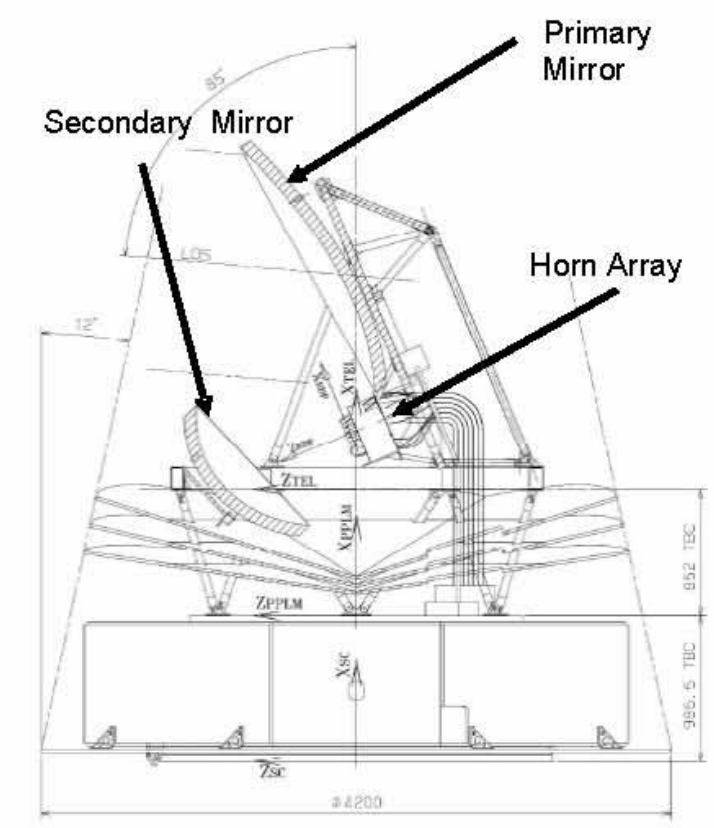

Figure 1. The Planck telescope layout showing the off-axis Gregorian configuration and the position of the focal plane arrays.

based on the use of scalar feedhorns fed by an overmoded waveguide [11] with the wavelength range selected by quasi-optically coupled mesh filters [12]. This gives an effective throughput greater than for a diffraction-limited system and maximizes the response to point sources.

The multi-mode channels are unique in their design and function and their development has necessitated the extension of existing modelling techniques to include both the analysis of overmoded corrugated waveguides [5, 13-15] and the propagation of partially coherent fields through the telescope optics and onto the sky [8,16-18]. The shaped horn configuration maintains control of sidelobes [11] and modifies the partially coherent illumination of the telescope. This has the advantage of increasing the sensitivity of the detection assembly, each extra mode contributing to the power detected. The drawback is the resulting beam characteristics are significantly more complicated to model and the boundary conditions are not as accurately controlled as for the single-mode channels because the precise balance of power in the modes is affected by the details of the feed relay system, which has the potential for cavity effects ([12, 19]).

A previous paper on the focal plane configuration was devoted to the design optimization and beam performances of Planck-HFI concentrating mainly on the CMB single mode channels [19]. This current paper is dedicated to the multi-mode channels of the HFI, ultimately focusing on beam pattern prediction for the channels at $545 \mathrm{GHz}$ and $857 \mathrm{GHz}$. Section 2 outlines the design solutions adopted to reach the specifications - including the requirements on illumination pattern of the telescope, edge taper and spillover levels. In section 3 the computational approach taken for the modelling of the multi-mode horns is described. Section 4 reports on how the horns reach the requirements in terms of telescope illumination and phase centre location and also comparison with the laboratory test measurements is discussed. Section 5 is dedicated to the extensive telescope 


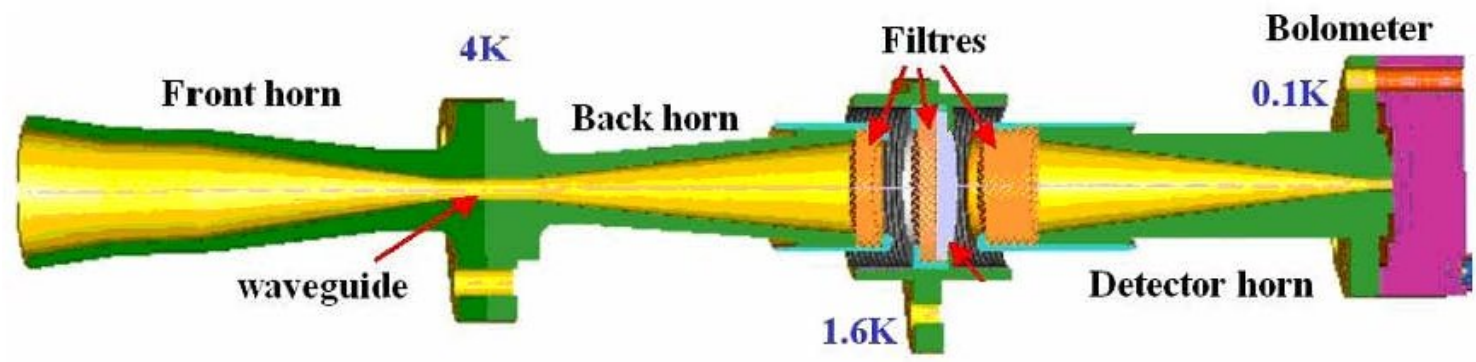

Figure 2. The typical optical chain for the HFI channels is shown schematically. The front horn determines the light collected from the telescope. It also strongly limits the sidelobe levels in the pattern illuminating the telescope reflectors. Its wave-guide section determines which modes couple to the detector. The back horn launches the CMB radiation through a set of filters into the $100 \mathrm{mK}$ horn that concentrates it onto the bolometer in the detector cavity. The precise dimensions of the horn assembly are shown in figure 3 for the $857 \mathrm{GHz}$ pixel. Note: the "Front" horn and "Back" horn assembly are referred to as the back-to-back (BTB) horn in the text of the paper.

beam simulations performed for the multi-mode channels based on the best expected realistically feasible performances of the horns and knowledge of the real in-flight telescope.

\section{Optical considerations and design constraints}

The Planck-HFI detection system is based on cryogenically cooled ultra sensitive bolometers [9] coupled to corrugated feedhorn antennas directly illuminating the telescope secondary mirror [1]. Shaped corrugated feedhorns (based on a previous geometry, see [20]) have been chosen for all channels to achieve very low sidelobe levels and strict stray-light control [12, 21]. There are no quasi-optical components in front of the horn aperture (as for example in the QUAD experiment [22]) which would have resulted in main beam distortion and necessitated a cold stop to terminate an overall increase in sidelobe levels [23]. Instead the front horn is part of a triple horn configuration in which the filters are located in between a back-to-back (BTB) horn and the detector horn (see figure 2, [12]). All of the waveguide sections are corrugated which for the CMB channels act as high pass filters defining the lower edge of the bands [15].

The design principle behind the triple horn configuration forming the detection assembly is presented in detail in Ade [12] and is based on a design concept by Church [24] (see also [25]). This arrangement is ideal for the single-mode CMB channels in that the optically coupled mesh filters will have no impact on the horn beam patterns. However for the multi-mode channels the components after the BTB waveguide can influence the detailed structure of the patterns and this needs to be considered and analyzed. Thus, since modelling to the required level of detail is impossible, especially as small tolerancing errors have a large effect, the multi-mode horn beams are less predictable in their actual performance than single-mode channels because of the propagation, scattering and potential reflections of multiple modes in the triple horn and cavity configuration. Nevertheless, the extra propagating modes in a multi-mode design improve the illumination pattern of the antenna, increasing the throughput and hence directivity. 
Table 1. Summary of optical requirements for each spectral band. Numbers within brackets refer to the goals.

\begin{tabular}{|c|c|c|c|}
\hline Band Central freq. & Target Resolution & Spillover (\%) & Edge Taper (dB) \\
\hline $545 \mathrm{GHz}$ & 5 arcmin & $0.5(0.3)$ & $-30(-32)$ at $26 \mathrm{deg}$ \\
$857 \mathrm{GHz}$ & $5 \mathrm{arcmin}$ & $0.5(0.3)$ & $-30(-32)$ at $27 \mathrm{deg}$ \\
\hline
\end{tabular}

Clearly Planck needs to be sensitive to faint foreground sources and so it is crucial to reduce unwanted signals to a minimum. Thus, the reduction of the spillover and the maximization of the power concentrated in the horn main beam are of high importance. Since the horns directly illuminate the telescope the horn beam sidelobes have to be minimized while the main beam fills as much of the telescope aperture as possible. In order to be consistent with the science requirements it was decided that the spillover has to be maintained to less than 1\% [4]. At the same time in order to prevent sidelobes due to truncation at the telescope mirrors the edge taper should be around $30 \mathrm{~dB}$. The design therefore needs to balance increased throughput and sensitivity to a point source while simultaneously meeting the spillover and edge taper requirements. The optical requirements as they relate to these Planck channels are summarized in table 1.

Coupling the horn directly to the telescope defines the optical characteristics of the Planck satellite (resolution, antenna main beam shape, sidelobe structure and spillover). For these high frequency multi-mode horns the balance between the modes propagating through the waveguide filter into the front horn determines the width of the beam illuminating the telescope and thus the spillover and edge taper. The balance between resolution and spillover depends ultimately on how the modes couple to the detector which is not necessarily a computationally well defined process. In modelling horns it has been assumed that optimal coupling to the bolometer is likely to be close to operational reality [19].

Although the sizes of the horn aperture and waveguide are driven by the optical requirements, there are also some mechanical limitations on the horn design as a result of their position at the centre of an array of the larger horn antennas for the longer wavelength channels (see figure 2). Each front horn has also been designed such that its phase centre is located on the telescope focal surface. The off-axis Gregorian telescope configuration (compensated aplanatic design to optimize the required field of view to accommodate the large focal plane assembly [1]) results in a focal surface with an off-centre apex. This shape has a pronounced slope across the focal surface. The high frequency horns are located in a line close to the centre of the focal surface where aberrational losses are least severe for the extended field of view. At this location the length of the high frequency horns was influenced significantly by the need to prevent shadowing by adjacent horns on the sloped focal surface (see figure 3). This implies tapered horns long for the operating wavelength band which turned out to be challenging to manufacture. The phase centre (determining location relative to the focal plane) was required to be within $4 \mathrm{~mm}$ of the horn aperture [26].

These manufacturing considerations forced modification of the original tapered horn profile of the back-to-back (BTB) section (as illustrated in figure 4 below), resulting in a more abrupt profile for the horn adjacent to the waveguide filter section. This has the potential to reduce somewhat the 

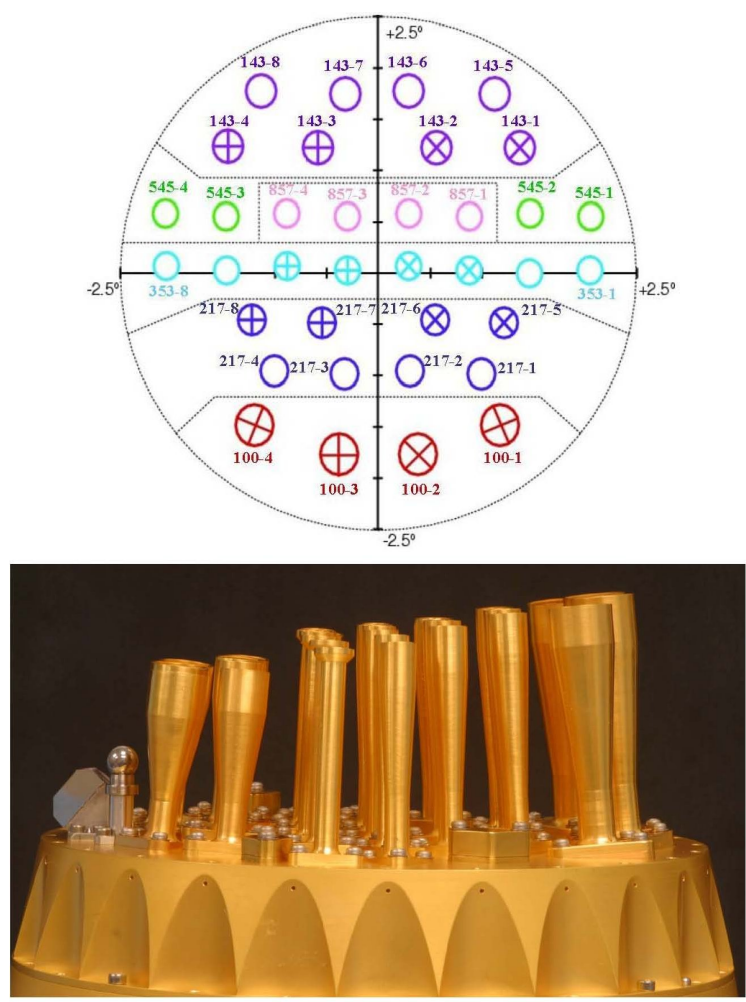

Figure 3. Focal plane layout of the HFI horns. The 545 and $857 \mathrm{GHz}$ horns are positioned where the optical quality is good enough for high frequencies (the $3^{\text {rd }}$ row from left in the picture). The curvature of rows compensates for the distortion of images by the telescope. The horns are designed so there is no shadowing by adjacent rows.

performance of the horn beam by increasing reflections from this part of the horn. A similar design was used for the detector horn. This effect was carefully analyzed to ensure no deterioration in multi-mode operation in terms of beam pattern and throughput occurred. In fact the beam patterns still achieve close to the desired requirements for sidelobe levels although the main beam is less tophat like in form (and thus the illumination pattern is somewhat less ideal, increasing the beamwidth marginally) and there are some more significant cavity effects due to the reflections in the system.

\section{Modelling of multi-mode horns}

In the high frequency $545 \mathrm{GHz}$ and $857 \mathrm{GHz}$ Planck pixels both the back-to-back (BTB) horn and the detector horn have overmoded waveguide filters (see figure 5). One can model the waveguidehorn structures using the same scattering matrix technique used for the single-mode-CMB-channel horns ([11]) except modes of all azimuthal orders $n$ that can propagate are included, not just those with $n=1$ ([5-8]). It is also assumed that in terms of the beam patterns reciprocity can be applied and that, in the quasi-blackbody cavity in which the bolometer is located, all waveguide modes are excited in power independently of each other so there is no phase relationship between them. Thus, many of these modes will contribute independently (i.e. incoherently) to the beam on the sky. A well designed single-mode horn transmits only one pair of orthogonal coherent fields, the $H E_{11}$ 


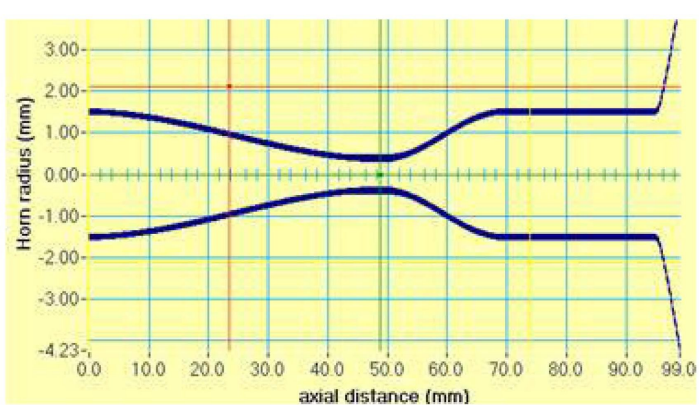

(a)

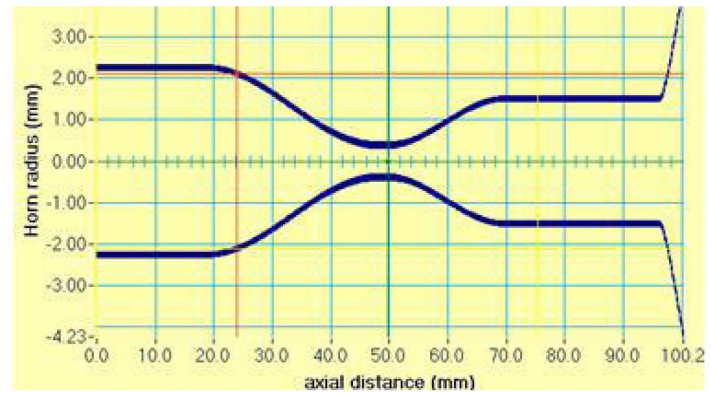

(b)

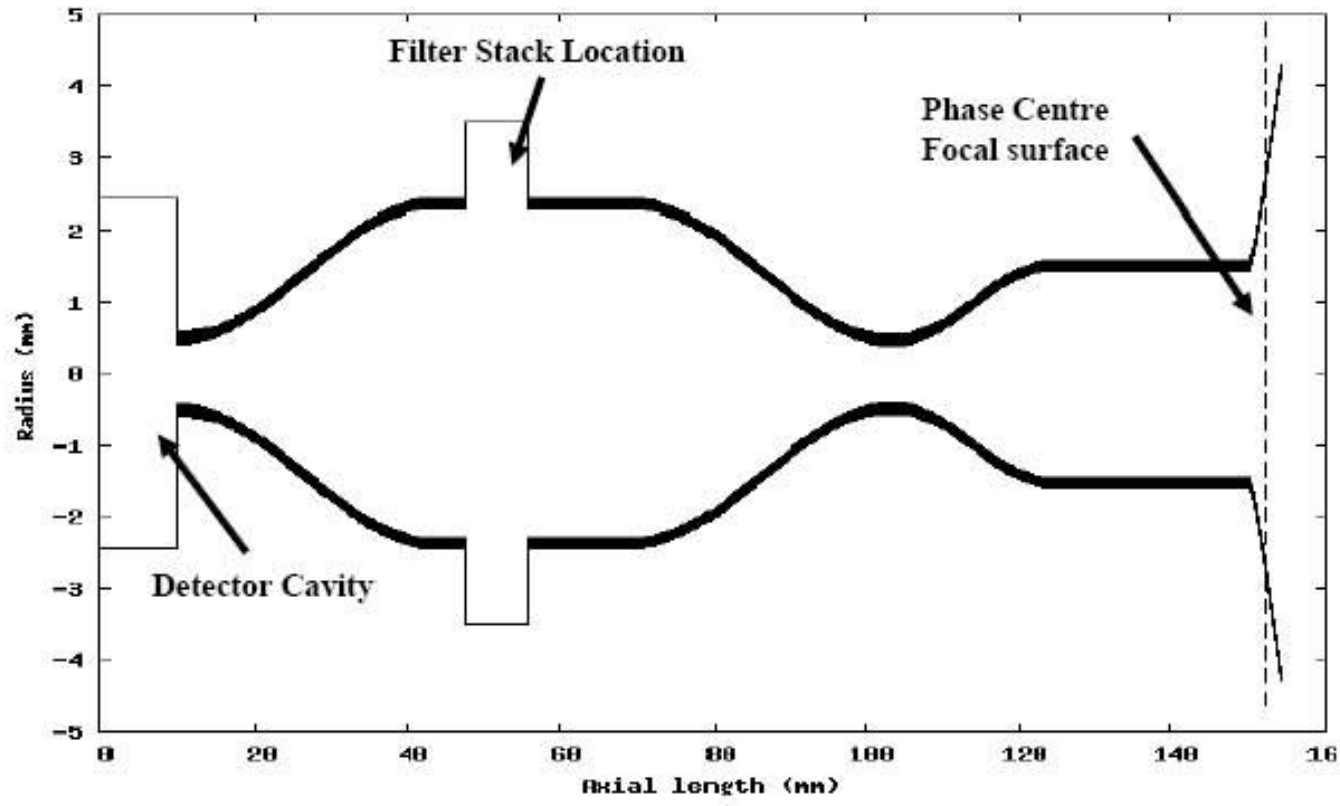

(c)

Figure 4. (a) Original profiled flared BTB concept. (b) Final manufactureable design. (c) Schematic diagram of the horn profile for full pixel simulations of a BTB plus detector horn (vertical scale significantly exaggerated). The locations of the detector cavity (schematic), filter stack (see also figure 2) and phase centre of horn (and focal surface) are indicated.

hybrid mode. In a multi-mode horn several independent pairs of orthogonal coherent fields will be present which will need to be propagated through the Planck telescope to predict the beams on the sky.

If there are a sufficient number of corrugations per wavelength the walls of the waveguide or horn can mimic a smooth continuous surface with a non-isotropic impedance. Four corrugations per free space wavelength are usually sufficient for this assumption [14]. The natural propagating modes of such waveguides are known as hybrid modes, as referred to above (pure transverse electric (TE) or transverse magnetic (TM) modal fields are not allowed). A circular corrugated waveguide is described by its radius and by the depth of the corrugations giving two parameters $r_{0}$ and $r_{1}$ (see figure 5). Within the context of the hybrid mode model these two radii will define the high frequency and low frequency cut-offs of the modes that can propagate in any waveguide section 


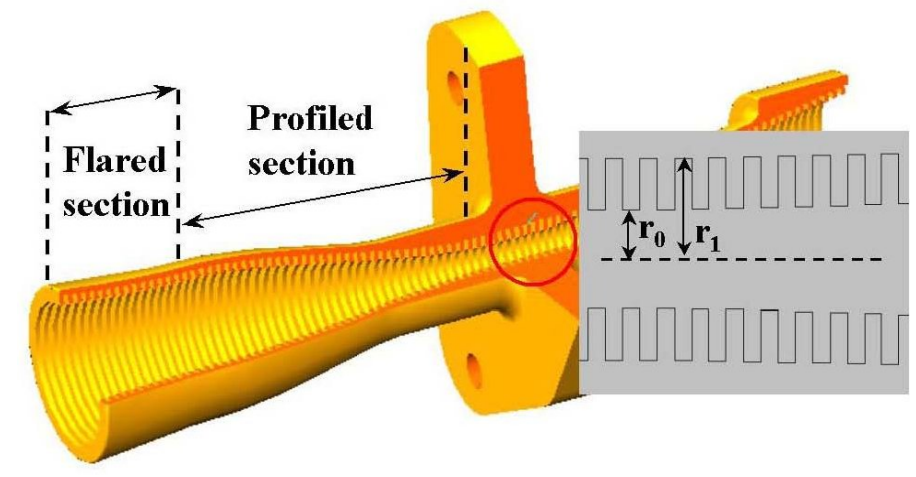

Figure 5. Section through horn showing the corrugations. The natural modes of such a system are hybrid waveguide modes $\left(H E_{n l}\right.$ or $\left.E H_{n l}\right)$. Such corrugated guides can be modelled as cascaded short smooth-wall segments as a band pass filter for hybrid modes.

forming a bandpass filter for each mode. Only a limited number of hybrid modes can propagate at any one frequency. The actual band of frequencies that can be transmitted is defined by the interference mesh filter stack [12] located between the BTB and detector horns. Note that different modes cut-on and cut-off at various frequencies across the photometric band (see figure 6). Thus at any given frequency only a subset of these modes will propagate. This approach gives a good understanding of the operation of the waveguide as a mode filter although it turns out to be less appropriate for the shaped horn where the relatively abrupt profiles results in significant mode scattering of these hybrid modes.

As an alternative one can regard the corrugated guide and profiled horn as a sequence of very short smooth-walled waveguide sections (representing sequentially the corrugation grooves and fins). For each horn segment the natural modes of propagation are the usual $T E_{n l}$ and $T M_{n l}$ modes of a smooth-walled cylindrical waveguide, with two possible orthogonal $T E_{n l}$ and $T M_{n l}$ fields for $n>0$ because of the cylindrical symmetry. At the junction between two segments significant scattering of power between modes occurs, which is kept track of using a mode matching technique such that complex power is conserved. By computing the scattering matrix terms for each junction one can compute the cascaded scattering matrix for the complete shaped waveguide horn structures. The details of the electromagnetic theory of multi-mode horn operation using this scattering matrix approach are given in a number of publications [5-8]. On propagation the family of modes with the same azimuthal symmetry scatter power into each other, but not to modes with a different azimuthal order $\mathrm{n}$, which simplifies the computation significantly. Also only modes up to a certain azimuthal order can propagate through the waveguide filter due to smooth-wall waveguide cut-off effects (see figure 6, for example).

Clearly in this discussion the term "mode" can be ambiguous, being both used within the context of the hybrid mode model and the mode matching scattering matrix technique with smoothwall waveguide modes as the "basis" set of fields. The hybrid mode assumption is valid if there are several corrugations per wavelength and if there is no abrupt change in the waveguide profile. The scattering matrix approach however is more accurate, particularly so as the number of corrugations per wavelength is lower than ideal for the high frequency $857 \mathrm{GHz}$ channels because of manufacturing limitations. At $990 \mathrm{GHz}$, the top end of the band, for example, there are only two 


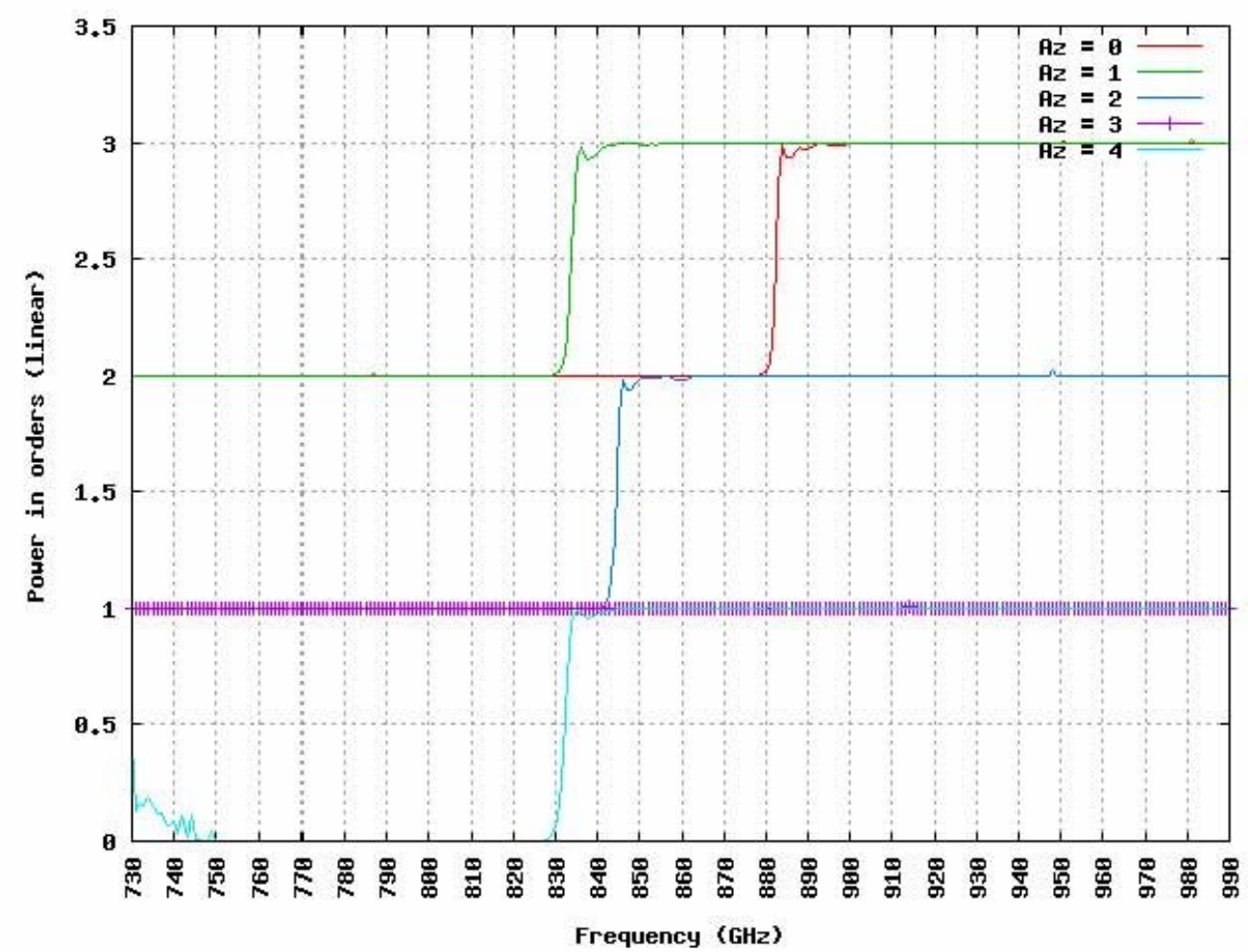

Figure 6. The plot illustrated how the power transmission (throughput) through the BTB horn increases with frequency for the different azimuthal families of modes. Each step corresponds to additional hybrid modes switching on (for example two of order $n=2$ switch on, whereas the number of order $n=1$ goes from two to three around $830 \mathrm{GHz}$ ).

corrugations per wavelength. Furthermore the shaped horns give rise to significant mode scattering even in the case of hybrid modes.

The mode matching approach has been shown to predict the beam patterns of single-mode horns to high accuracy and reproducibility [11]. The profiled-flared horn design (figure 2) was also used on the earlier experiment Archeops (a CMB experiment to test the Planck concept [27]) to produce the required beam shape. The carefully optimized hyperbolic flare section reduces the sidelobes surrounding the main beam to acceptable levels (the flare length chosen was a compromise between optimized sidelobe reduction, beam illumination pattern and the necessity to place the horn phase centre close to the horn aperture). Subsequent analysis shows that it is the presence of the correct amount of the $H E_{12}$ mode at the horn aperture which provides the sidelobe reduction [28]. Note that while the phase centre of a profiled horn is located close to its aperture, the addition of the hyperbolic flare moves it further inside the new aperture, the precise location depending on the length and the tilt of this flare and the frequency. This effect had to be taken into account when designing the horns in order that they met all the optical requirements and thus were at the correct location with respect to the focal surface not to shadow, or be shadowed by, the other horns.

At the front aperture of the BTB one can recover the true independent hybrid fields that are transmitted by the waveguide filter and horn. These hybrid fields propagate independently through 
the telescope and onto the sky since they were generated by an independent set of smooth-wall waveguide modes at the effective black body enclosure at the detector [11, 14]. The columns of the $S_{21}$ scattering matrix contain the mode coefficients for the modal sums required to recover the independent hybrid fields at the horn aperture. These fields, once recovered, must be propagated to the sky independently and then be added in quadrature to simulate a true multi-mode beam pattern, each simulation being at a single frequency. In order to produce a true broad band beam these single frequency intensity patterns must then furthermore be added across the band with any nonuniform weighting to take into account any non-uniform transmission for the filters that define the band. The spectrum of the source will also affect the beam pattern for any particular observation.

An important issue is how well modes in the cavity make it through the detector horn waveguide filter relay and couple to the BTB horn. Since the waveguide filter in the detector horn is wider than that of the $\mathrm{BTB}$, it is a reasonable working assumption that any mode that can propagate through the BTB also propagates through the detector horn waveguide from the cavity. Thus, as far as the beam patterns are concerned a useful first order working model is to assume that the BTB is effectively coupled to a black body cavity (the detector cavity). For the modelling of the horns this translates as the assumption that all modes used in the finite mode model are excited at the back end of the BTB horn independently and with equal power. This was justified on the basis that the cavity is designed to act as a black body over the operating band, and that measurement of beam patterns for single mode horns is in good agreement with the models. It is a useful approach in the design of the horns for optimising the illumination patterns on the telescope. On the other hand for the simulations of the telescope beam patterns on the sky described in section 5, models of the complete pixel assembly were used, not just the BTB horn. These complete pixel models showed much more complex scattering behaviour than the BTB models, in qualitative agreement with the scattering behaviour observed in laboratory measurements. It is possible to spectrally bias at the level of individual frequencies after deriving the beams on the sky, assuming the cavity is a grey-body whose spectrum is known. Likewise the attenuation in the filter can be taken into account.

All the back-to-back high frequency horns of HFI went through qualification and characterization tests at single component level. The result of their transmission measurements is reported in another publication [17], which includes beam pattern measurements outside of the normal operating band at a frequency at which the horns were single-mode. The horns show very little deviation from the ideal pattern (model prediction for single mode operation) at the level of their main beam (+/- 25deg corresponding to the edge taper) indicating the absence of obvious manufacturing defects likely to affect the beam patterns. The measurements of the beam patterns made at multi-mode operation are discussed below in section 4 .

\section{Telescope illumination patterns and laboratory tests}

The simulated broadband far-field patterns of the 545 and $857 \mathrm{GHz}$ horns are shown in figure 6 below. In figures $6 \mathrm{a}$ and $\mathrm{b}$ black-body illumination of the front BTB horns is assumed, equivalent to perfect coupling through the relay feed system to the detector. The beams for the $857 \mathrm{GHz}$ horn are shown in figure $6 \mathrm{a}$ at spot frequencies at the band edges $(730 \mathrm{GHz}$ and $990 \mathrm{GHz})$ and centre $(857$ $\mathrm{GHz}$ ) illustrating that the beam width remains approximately constant (throughput conservation). Similarly, the $545 \mathrm{GHz}$ horn beams are shown at the band centre and the edges (figure 6b). 


\begin{tabular}{|c|c|c|}
\hline \multicolumn{3}{|c|}{ Spillover and edge-taper levels } \\
\hline Average Values & $545 \mathrm{GHz}$ & $857 \mathrm{GHz}$ \\
\hline Spillover $(26 \mathrm{deg})$ & 0.0056 & 0.0011 \\
\hline Edge Taper $(26 \mathrm{deg})$ & $-26.0 \mathrm{~dB}$ & $-29 \mathrm{~dB}$ \\
\hline
\end{tabular}

Table 2. Spillover and edge-taper levels.

A more complete model of the complete Planck pixel, including the detector horn and transition to the bolometer cavity, has also been investigated, as discussed above. Two assumptions were made here in modelling the full BTB plus detector horn assembly: (i) the filter section could be described by a smooth waveguide section of the same diameter as the mesh filters and (ii) the bolometer cavity itself is approximated as a radiating black body source or sink (i.e. there are no reflections). An exact representation of the pixel was not possible because of the nature of the quasi-optical coupling of the filter section. To overcome this difficulty a number of example cases were considered, in which further simulations were undertaken, to quantify the effects on the beam patterns of the BTB resulting from the inclusion of the cavity, detector horn and the filter-assembly in the analysis. With the help of these varied theoretical techniques, in-flight performance can be estimated (see section 5 for further discussion).

Comparison of the plots (see figure 6) shows that the detector horn and filter section act as a good relay for coupling to the BTB horn so that the assumption of blackbody illumination of the front BTB horn would appear to be reasonably valid (typically close to $100 \%$ level for propagating hybrid modes). In any case in the determination of the beams on the sky the complete far-field Planck pixel illuminations of the telescope were used. Note that the edge tapers in all cases satisfy the requirement of better than $-30 \mathrm{~dB}$ at 26 degrees at the centre of the band. The spillover values for the various horns are given in table 2 .

Determining the so called phase-centres of multi-mode horns requires careful consideration. The "phase centre" is an ill defined concept as the individual spatially coherent fields making up the far-field patterns all appear to come from different phase centre locations inside the horn aperture. Finding the best location at which to place these horns with respect to the focal surface therefore requires a different approach from that of a single mode horn [11].

In this case it is reasonable that the average phase centre location is best defined as at the waist of the beam obtained by propagating backwards into the virtual space behind the horn aperture (see figure 8). Since the phase fronts of the modal fields at the horn aperture are convex, the effective beam waist "phase centre" will be located inside the horn. The focus of the telescope should be located at this position in order to optimize the angular resolution and on-axis gain for the beam on the sky [26]. However, because of the multimode nature of the beam, the exact position where the on-axis gain is a maximum may not coincide precisely with the optimized angular resolution. Note: the individual spatially coherent component fields must be propagated separately and combined in quadrature to determine the intensity pattern. The waist position is also frequency dependent clearly.

The effects of telescope truncation can be neglected in determining the position of the best phase centre as, in Planck, the beam edge tapers at the telescope are very low (about $-30 \mathrm{~dB}$ ) and to 


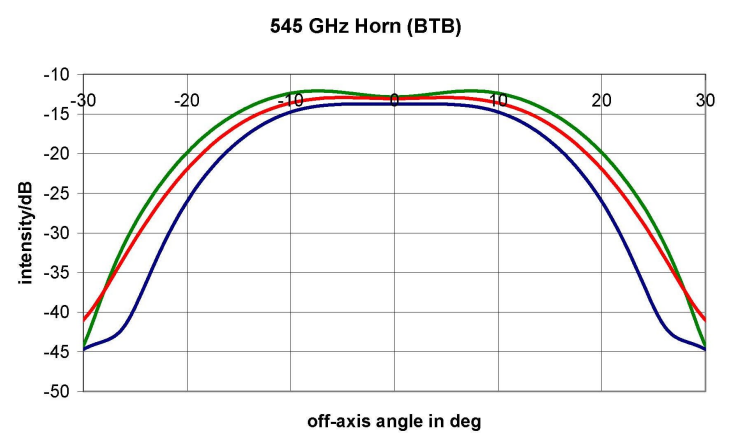

(a)

$645 \mathrm{GHz}$ Horn (BTB)

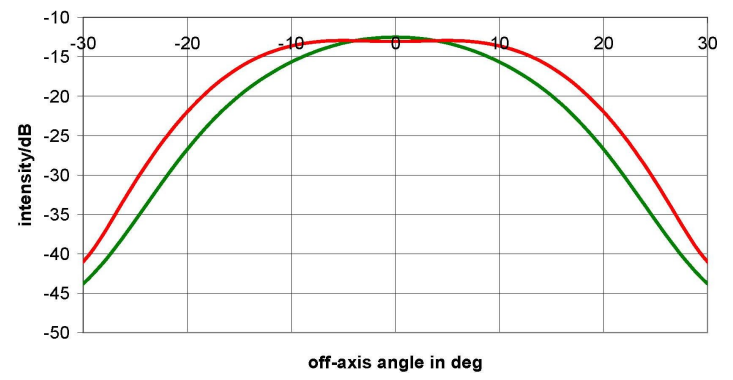

(c)

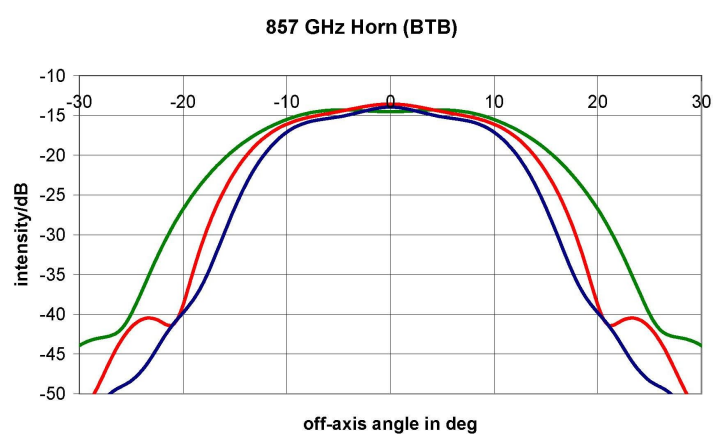

(b)

$857 \mathrm{GHz}$ Horn (BTB)

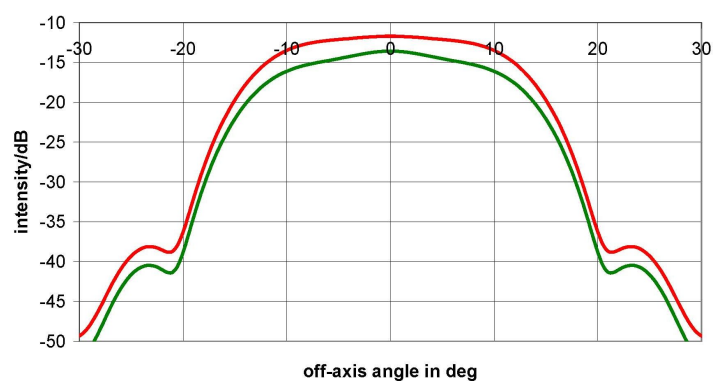

(d)

Figure 7. (a) Comparison 545 BTB far-field horn beam patterns at band centre and band edges. (Green465, Red-545 GHz, Blue-625 GHz). (b) Comparison 857 BTB far-field horn beam patterns at band centre and band edges. (Green-730 GHz, Red- $857 \mathrm{GHz}$, Blue-990 GHz). (c) Comparison of far-field horn beam patterns at band centre for $545 \mathrm{GHz}$ horn for BTB (red) and full pixel cases (green). (d) Comparison of far-field horn beam patterns at band centre for $857 \mathrm{GHz}$ horn for BTB (red) and full pixel cases (green).

a good approximation the telescope can be assumed to be of infinite extent. Thus the field on the sky will be an image of the horn field at the telescope focus without much broadening because of the low level of spatial filtering at the telescope aperture. Aberrations degrade the resolution only slightly.

The depth-of-field will be affected by the spatial filtering of the telescope. A realistic estimate for these horns is obtained by including the effects of the edge taper at the telescope, which thus involves coupling the far-field of the horn to the telescope and defocusing the system by adjusting the distance between the horn and telescope, effectively changing the phase curvature of the beam at the telescope aperture. Replacing the telescope with an equivalent paraboloid, the radiation pattern on the sky is obtained by summing in quadrature the Fourier transforms of the far-fields of the independent spatial modes of the horn with the appropriate phase error across the telescope aperture. In this case one can then analyze the variation of the on-axis gain on the sky with defocus distance as a convenient parameter for checking the depth of field on the focal plane.

Figure 9 shows the broadband on-axis gain of the 545 and $857 \mathrm{GHz}$ horns with horn aperture displacement away from the focal plane. A negative distance implies that the focus is positioned behind the aperture (where it is expected). The maximum on-axis gain occurs when the telescope focus lies approximately $2 \mathrm{~mm}$ behind the horn aperture, thus satisfying the Planck requirement that the phase centre must be no more than $4 \mathrm{~mm}$ from the aperture to prevent shadowing of other 

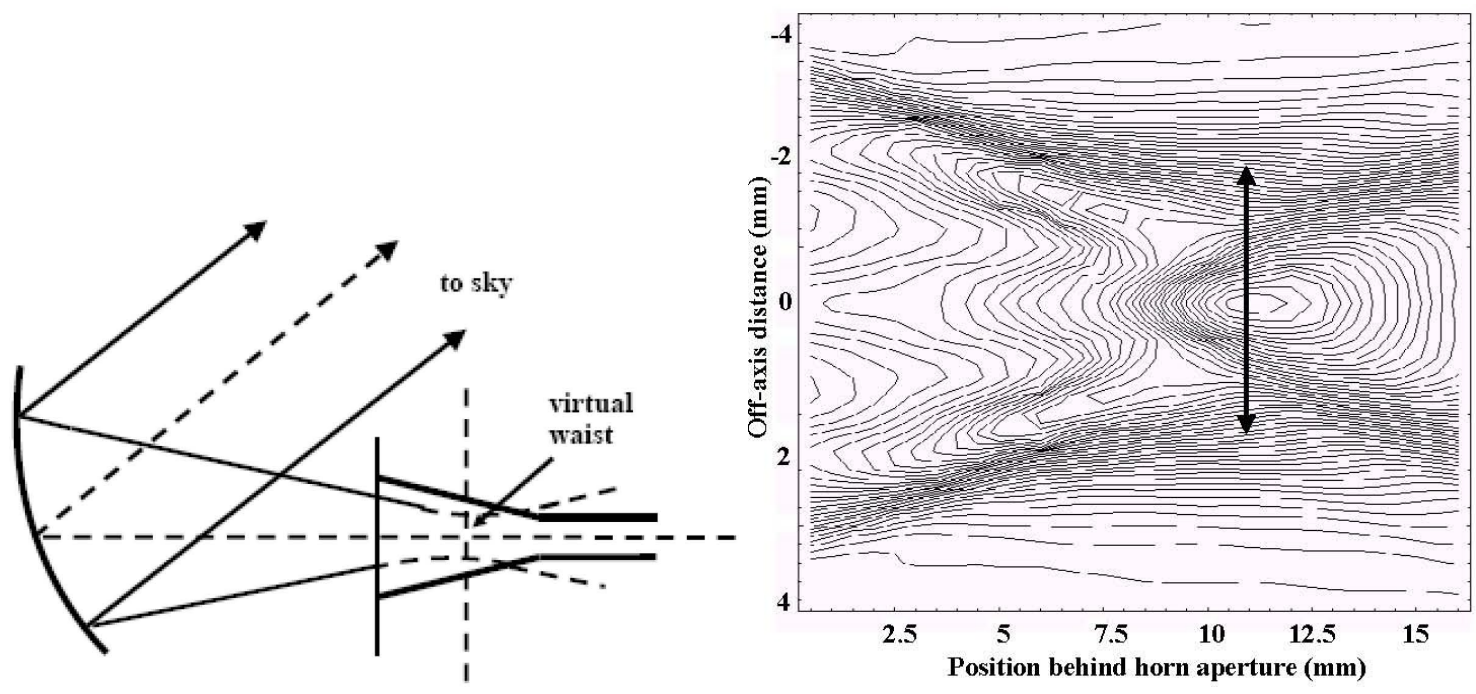

Figure 8. Schematic of set-up of a horn with respect to an antenna illustrating the horn virtual waist coinciding with the antenna focal point. Typical behaviour of the beam in the vicinity of the virtual waist location is also illustrated.

horns in the HFI and LFI arrays. For both horns the depth of field (whose edge is defined by the $1 \mathrm{~dB}$ level below the peak level) is about $10 \mathrm{~mm}$ in both cases indicating a rather tolerant system to axial positional inaccuracies and satisfying all phase centre requirements.

The special operation in space of the multi-mode Planck pixels with unimpeded illumination of the telescope and low temperature of the optical elements meant that an exact measurement of the optical response could not be made under flight conditions. Instead on-ground characterization of these pixels has been based on multiple measurements of qualification and flight model feedhorns. Initially the operation of overmoded BTB horns was investigated using scaled prototypes at $150 \mathrm{GHz}$ to verify theoretical techniques for modelling such horn systems and good agreement was found between theory and measurement [7]. Furthermore the flight model BTB horns were tested for single mode operation at a frequency below the actual band of operation to check for manufacturing defects. Again good agreement between prediction and measurement was recorded in these cases. Of course under single mode operation the beam shape is unaffected by the part of the pixel behind the BTB waveguide filter.

The shear complexity of the measurement meant it was not possible to test the complete flight model pixels for the multi-mode channels in the laboratory. However, measurements were made at Cardiff University of a multimode horn assembly at $857 \mathrm{GHz}$, which did include a flight model Planck BTB horn (see figure 10b). However, the arrangement is different from the actual flight configuration of a complete Planck pixel. The most sensitive measurements were obtained by placing the cryostat window behind the flight model Planck BTB horn (so it was external to the cryostat) and also introducing an extra relay BTB horn section (parabolic horns) between the detector horn and the cryostat window [29]. The $857 \mathrm{GHz}$ broadband and spot frequency beams measured for this assembly were narrower than those predicted for a full Planck pixel arrangement. Figure 10a shows the results of the measurements made over the band defined by the Planck filters. We also overlay the broadband prediction for the $857 \mathrm{GHz}$ full pixel (which is clearly wider). 

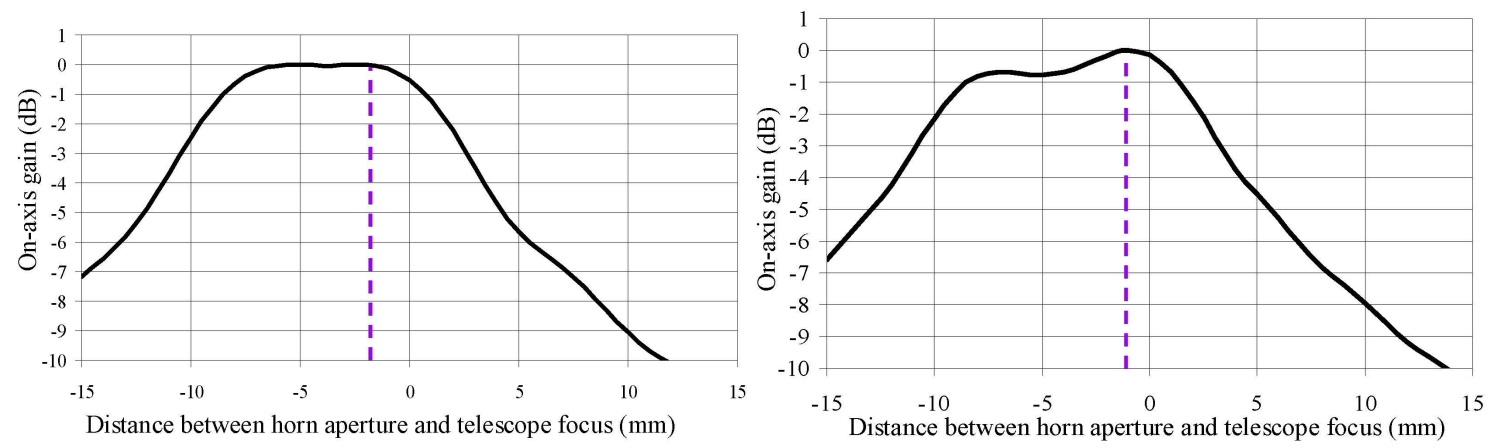

Figure 9. Broadband on-axis gain in the far-field of a perfect telescope for varying displacements of the horn aperture with respect to the telescope focus for the $545 \mathrm{GHz}$ (left) and $857 \mathrm{GHz}$ (right) horns. Optimum gain lies between $2 \mathrm{~mm}$ and $7 \mathrm{~mm}$ behind the horn aperture.

The discrepancies with the Planck pixel are understandable when the measurement set-up is considered, particularly the necessity to include the extra relay BTB in the assembly with a mismatch at the Planck BTB horn. This mismatch leads to extra mode filtering. Also, it was not possible to model the system precisely or to the level of accuracy of the Planck pixel configuration because of the presence of the cryostat window in the measurement set up and the long air-gap between the parabolic BTB horn pair and the Planck BTB pair. When one considers the mismatch between the diameters of the horns on either side of the cryostat window and the field patterns at the horn aperture for the various azimuthal orders, we expect significantly reduced transmission for modes with off-axis field distributions (modes for which $n \neq 1$ ). Thus, an approximate analysis suggests that the beam will be more dominated by the $n=1$ azimuthal modes, with some power at $n=0$ and possibly $n=2$. By adjusting the levels of these modes a much better fit to the data is obtained. The analysis of this system is considered in further detail in Ade and Savini [29].

The narrower beams measured of course also indicate fewer modes propagating compared with the full Planck pixel. This is also suggested by the throughput, as if one assumes $A \Omega=$ $n \lambda^{2}$, then, $\Omega_{\text {meas }} / \Omega_{\text {full-pixel }}=n_{\text {meas }} / n_{\text {full-pixel }}$ giving a $20 \%$ to $30 \%$ loss in measured beam area, corresponding to a $20 \% / 30 \%$ loss in the number of modes. Thus, the horn assembly is clearly working in a multimode fashion and it is possible to show that the partially coherent fields can be re-expressed as a sum of hybrid fields. Contributions to the beam can also be grouped according to azimuthal order and overlaid with the measurement data to investigate which field distributions are missing. As noted above modes not of order 1 appear to be significantly reduced and if only modes of azimuthal order $n=0$ and 1 are added together, the resulting beam pattern can be fitted to the measured beams to a reasonable approximation by scaling the input mode amplitudes (see figure 11). This is not a unique solution of course, given the approximate analysis, but is strongly suggested by the fact that the higher azimuthal orders are more extended. The noise level at $-22 \mathrm{~dB}$ also makes it difficult to determine the contributions of the more extended modes.

In the case of the in-flight behaviour of the Planck pixel, beam patterns are expected that have wider beams than given by the laboratory measurements on the assembly described. Modelling of the full Planck pixel indicates that without the extra relay BTB or Cryostat window there will now be no significant attenuation of the modes making up the multi-mode distribution through the flight 


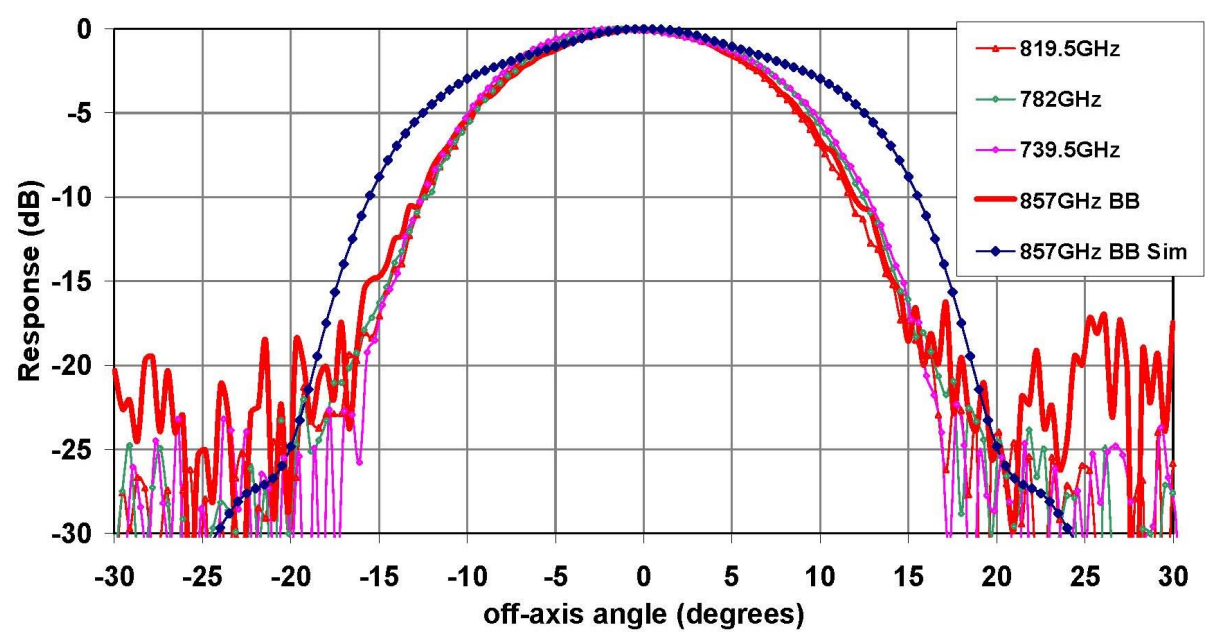

(a)

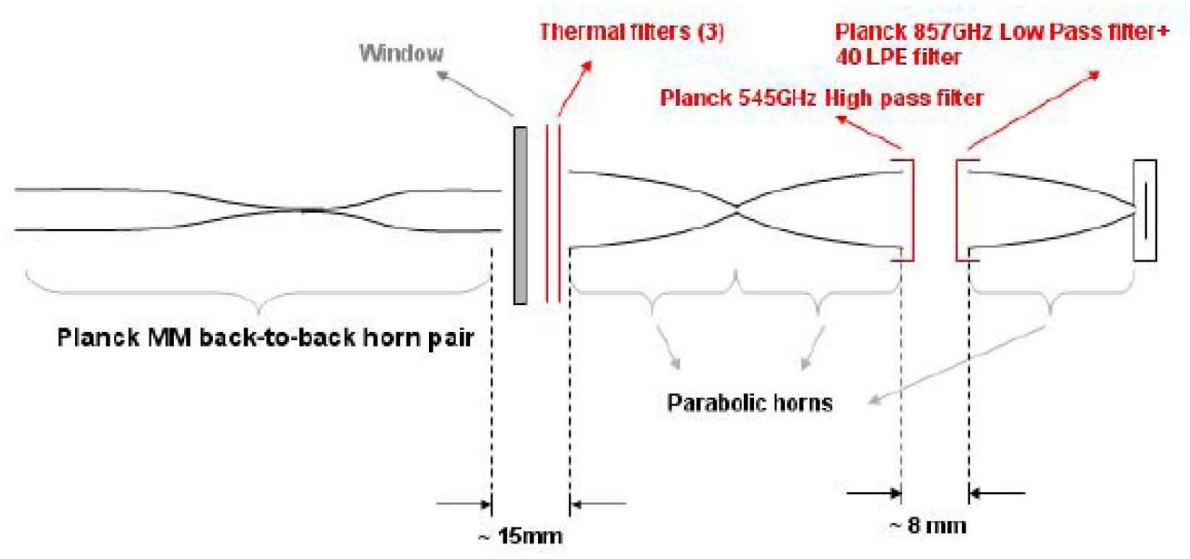

(b)

Figure 10. (a) Horn far-field measurements made at Cardiff for broadband and spot frequencies with the BTB horn outside the test Dewar window. The " $857 \mathrm{GHz}$ BB sim" case refers to the broadband simulations of the BTB horn assuming a perfect Planck pixel configuration. Simulation of the test set-up assuming mode filtering is shown separately in fig 11 for clarity. (b) Schematic of the $857 \mathrm{GHz}$ horn assembly at Cardiff with the BTB horn outside the test Dewar window.

pixel. The presence of the window and the long air gap implies only an approximate calculation was possible. In the next section we consider the impact of these issues on the telescope main beam patterns on the sky and the angular resolution of Planck.

\section{Telescope main beam patterns}

Realistic beam patterns on the sky require realistic models of the horns. The simulation of the full horn assemblies themselves has been a revealing and challenging undertaking, particularly when including the coupling to the detector horn in the simulations. To perform these simulations of the multi-horns it has been necessary to develop a computationally efficient modelling program. The code performs run-time checks on the numerical stability of the scattering model at each junction and basic checks on the physical plausibility of the model. With the new software the pixel assem- 


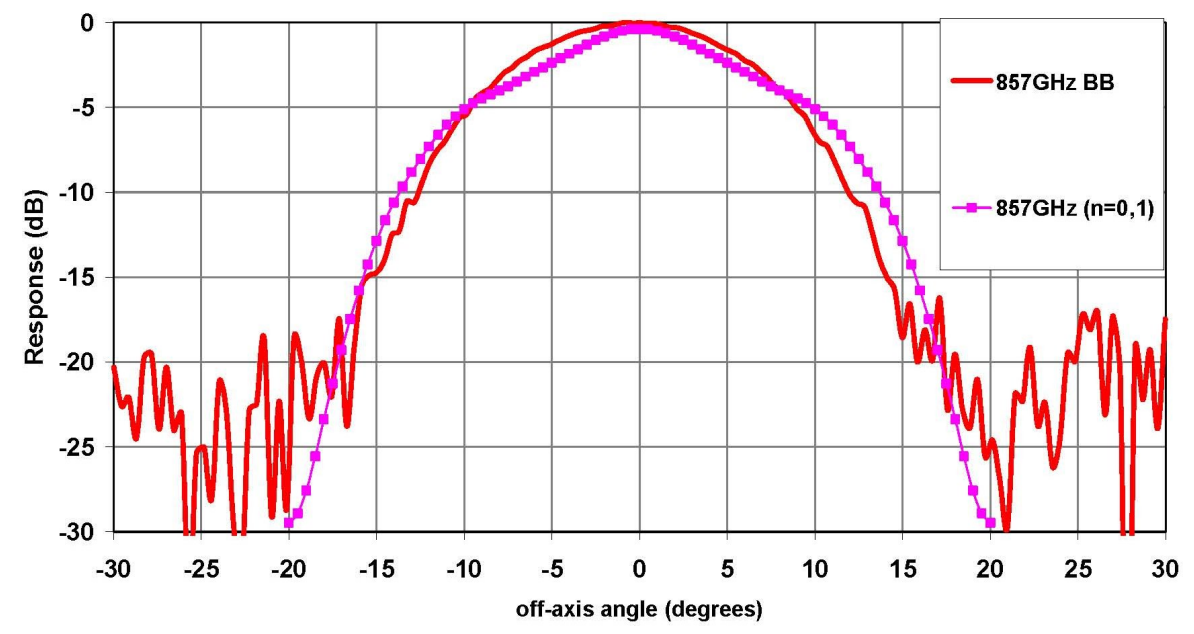

Figure 11. Far field of the 857 BTB horn with reduced mode content (azimuthal orders $n=0 \& 1$ only) overlaid with the data taken at Cardiff University (both normalised to $0 \mathrm{~dB}$ on axis).

bly could be simulated in $1 \mathrm{GHz}$ steps across the band for a number of models. This has been done for the ideal (as designed) horn and, in the case of the $545 \mathrm{GHz}$ horn, for eight models of horns with manufacturing tolerances not exceeding $2 \mu \mathrm{m}$ applied on a section-by-section basis to see if there was any observable effect. For the $857 \mathrm{GHz}$ horn the effect of manufacturing tolerances has been examined for eleven models at a number of spot frequencies.

The more realistic models presume a perfect filter in front of the detector horn, and the working assumption is that the bolometer cavity is a perfect black body across the band. The horn models do not take into account assembly imperfections that would result in non-perfect alignment of the sections. Misalignment would give rise to the exchange of power between fields of different orders, effectively giving rise to leakage of power into non-propagating fields. It is not immediately obvious what the effect of misalignment would be, but it would probably result in a very small reduction in throughput and some damping of any power trapped due to standing waves in the system.

Overall the performance of the complete horn assemblies is similar to that of the BTB horn alone, with a reduced throughput, but a similar change in beam pattern across the band. The modelling indicates that there is trapped power between the bolometer horn and the BTB horn, the horn configuration acting as a leaky cavity (see figure 2). This gives rise to rapid frequency dependent changes in throughput. Furthermore, as well as total power transmission variations, there is frequency dependent variation in the distribution of power between fields of all orders. That means that there are beam pattern changes with frequency that would not occur in the BTB horn. Because it is not possible to know what has been built to the level of accuracy to which the horn is sensitive, it is not possible to know precisely what the beam pattern will be in very narrow bands. Nevertheless, over any $15 \%$ sub-band of the spectral band this 'ringing' tends to average out, and the net effect is only a reduction in throughput. Within a narrow band variations of up to $10 \%$ are seen.

Inclusion of damping due to finite conductivity does not materially affect the qualitative behaviour observed. Single frequency or very narrow-band beam pattern prediction should be considered only as a qualitative indicator of performance. This is because very small manufacturing 
tolerances change the distribution of power between transmitted fields in much the same way as a change in frequency. As a result, the qualitative behaviour of two tolerance models of the broadband horn will be much the same, but the distribution of power between modes and the mode-bymode transmission of the different horns will be different at any one frequency.

Conclusions made above are also supported by analysis of the lower frequency $100 \mathrm{GHz}$ HFI horns. In this case it was found that, with an approximate model of the full $100 \mathrm{GHz}$ pixel assembly, although 'ringing' is seen, the effect is weak with almost unity power transmission across the band dropping off sharply below $86 \mathrm{GHz}$. Thus ringing is only a significant feature for multi-mode horns.

The simulations of the telescope beam patterns for the multi-mode horns were undertaken using the Physical Optics with the Physical Theory of Diffraction method implemented in the GRASP software package (www.ticra.com). A model of the telescope at the operating temperature of $45 \mathrm{~K}$ was built in the GRASP package using the measurements of the telescope. The source fields that were to be propagated through the GRASP telescope model were sets of coherent horn aperture fields. A separate set of aperture fields was derived from mode-matching models of the complete pixel assemblies at evenly spaced frequency steps across the pass band for each of the 545 and 857 $\mathrm{GHz}$ horns. Many frequency steps were used to represent the radiated field of the horns because, unlike the BTB horns on their own, the scattering within the complete pixel assemblies is complex and gives rise to an aperture field and power transmission that is sensitive to the frequency.

Since the detectors are wideband and the GRASP calculations are monochromatic, several spot frequency patterns need to be calculated and averaged to simulate the beam pattern, with each calculation repeated for each orthogonal polarization. For characterization of each main beam single polarization several frequencies were calculated across the band. For multi-mode horns the number of modes transmitted to the bolometer varies with the frequency. The resulting fields are incoherent sums of all the modes present and the beam pattern varies with frequency. Analysis of the scattering models of the $857 \mathrm{GHz}$ horn aperture fields indicates that there are essentially only a small number of independent hybrid mode fields contributing to the beam on the sky. Modelling the telescope beams involves identifying those hybrid aperture fields which have significant power (greater than $0.1 \%$ of the total power) and independently propagating them through the telescope one at a time for spot frequencies across the band, then adding in quadrature.

The broad-band beam for the $857 \mathrm{GHz}$ horn was modelled between $730 \mathrm{GHz}$ and $990 \mathrm{GHz}$ in $5 \mathrm{GHz}$ steps; a total of 53 frequencies (see figures 12 and 13). The model of the horn used 64 modes in each azimuthal order 0 through to 4 ; there is no transmitted power in higher azimuthal orders. Thus, at each frequency the horn model has an input of 320 waveguide modes of unit amplitude, the output being a frequency dependent number of aperture fields. In all, 743 aperture fields were found to be sufficient for a good description of the broad-band beam, sufficient meaning that the addition of further frequency contributions makes a change in the field patters smaller than the modelling error of approximately $-60 \mathrm{~dB}$ below the peak. Each of these fields is coherent and has to be propagated independently through the telescope model to the sky. Having calculated the beam pattern on the sky for each aperture field they are summed in quadrature. This process gives a broad-band beam power density pattern with units W/sr (the units used by GRASP for far-fields).

The $545 \mathrm{GHz}$ broad band telescope beams have been simulated taking the same approach as that described for the $857 \mathrm{GHz}$ beams. The beam pattern shown in figures 12 and 13 are derived from 63 frequency contributions. 

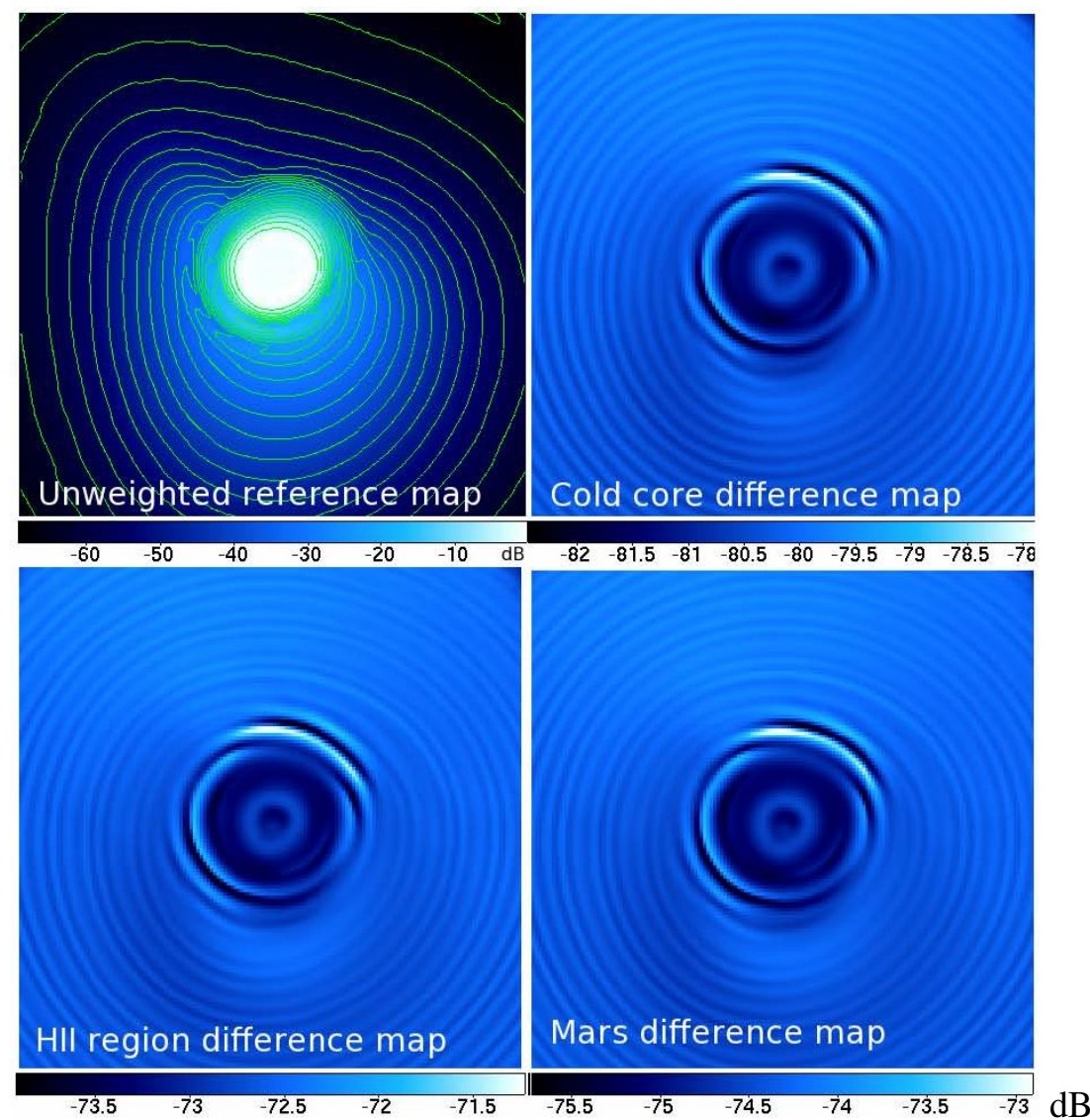

(a)

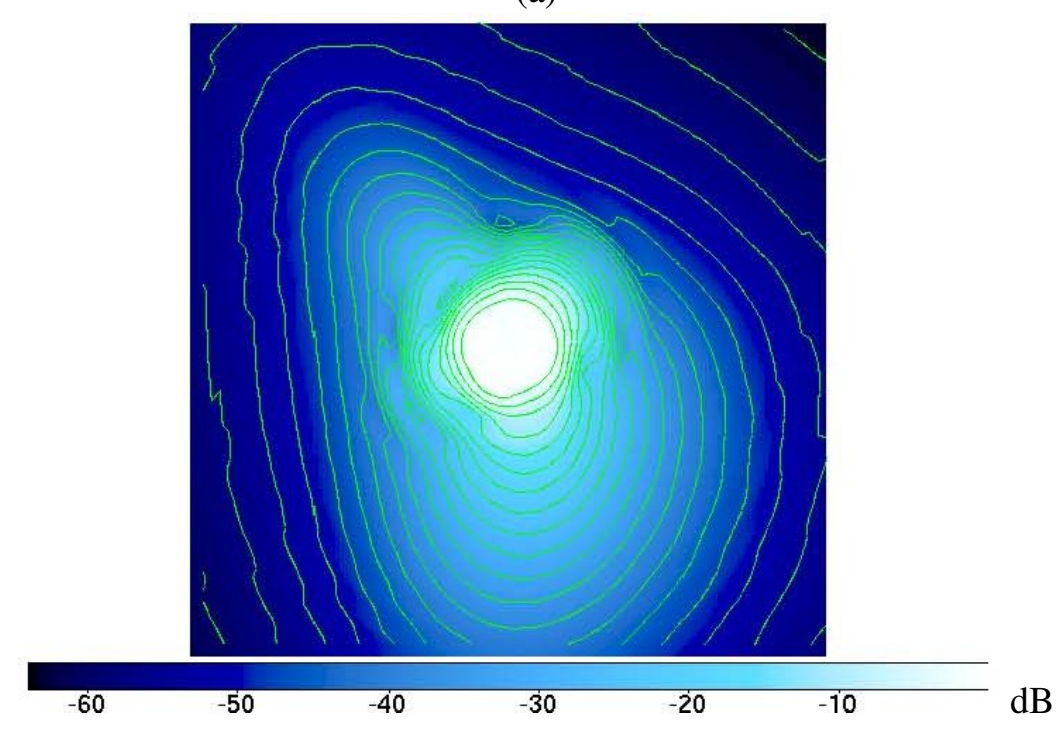

(b)

Figure 12. (a) H-857-1 unweighted reference beam and difference maps for the spectrally weighted $730 \mathrm{GHz}$ to $990 \mathrm{GHz}$ broad band beam patterns. Contour levels are at $3 \mathrm{~dB}$ intervals. The maps cover $30 \times 30$ arc minutes. (b) H-545-2 beam pattern for $465 \mathrm{GHz}$ to $630 \mathrm{GHz}$. Contours are at $3 \mathrm{~dB}$ intervals. The maps are in Ludwig UV coordinates covering $30 \times 30$ arc minutes. 


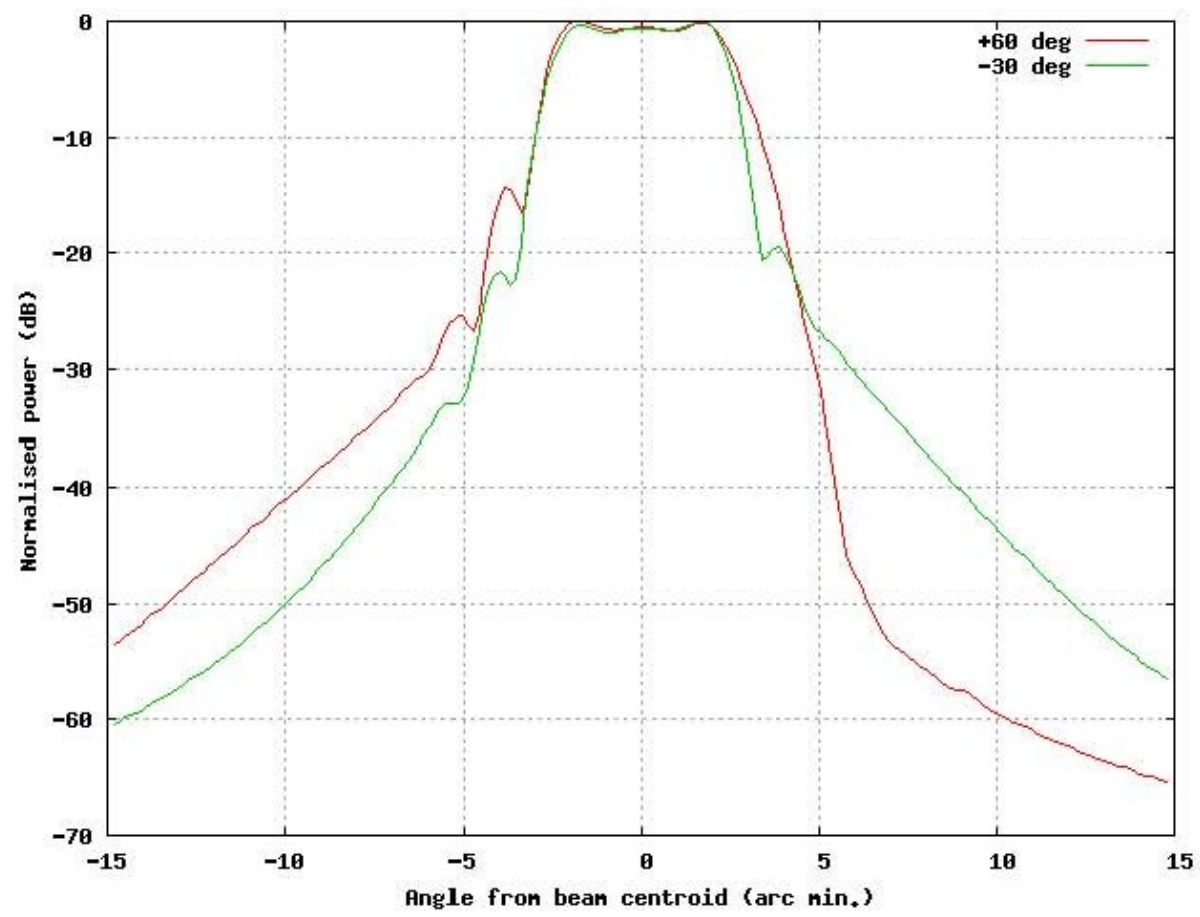

(a)

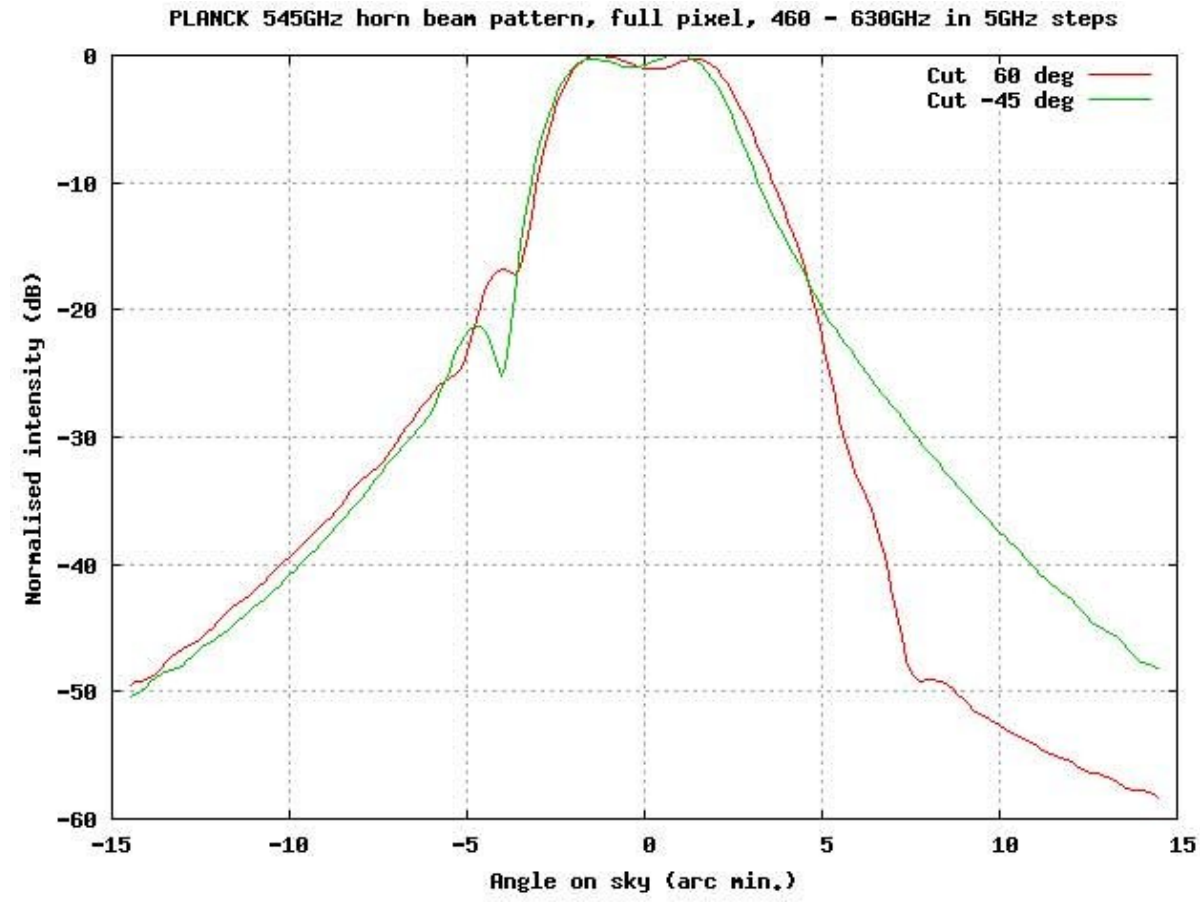

(b)

Figure 13. (a). Orthogonal cuts through the H-857-1 beam. (Normalised power in dB.) (b)Cuts through the H-545-2 beam. (Normalised power in $\mathrm{dB}$.) The cuts are in the scan and cross-scan directions for the Planck satellite. 
Note that since the main beam will not be Gaussian, being made up of many modes, a classic Full Width Half Maximum (FWHM) used on a typical quasi-Gaussian main beam becomes an inappropriate measure of the beam width. Examples of such beams are shown in figures 12 and 13 for the full pixel detection assemblies computed across the spectral bandwidth (broadband). The main beam is clearly non-Gaussian and the usual value of the FWHM becomes very sensitive to on-axis gain. A better measure of the beam width is to use encircled energy and define the encircled energy beam width (EEBW) to be the radius of a circle including one half of the total power in the beam.

Estimates for the FWHM and EEBW are obtained by taking two beam cuts through the centroid of the unweighted beams in the widest and narrowest directions. For the H-857-1 pixel the beam is slightly elliptical and rotated through approximately 30 degrees. The estimates for the beam without any spectral weighting are: FWHM: $5.051 \times 4.835$ arc min; EEBW: $3.668 \times 3.448$ arc min. Figure 13a shows the two orthogonal cuts through the spectrally unweighted beam. Spectrally weighting the beams for Mars, Sirius, HII regions and cold cores changes the beam pattern so little that it is not observable on a plot except upon very close examination (see figure 12a for the difference maps). The broad-band beams are therefore not spectrally biased.

The broad-band beam pattern FWHM and EEBW estimates for the pixel H-545-2, based on calculations for the frequency range 465 to $630 \mathrm{GHz}$ in steps of $5 \mathrm{GHz}$, are: FWHM: $4.85 \times 4.69$ arc min and EEBW: $3.74 \times 3.36$ arc $\min$ (see figures $12 \mathrm{~b}$ and $13 \mathrm{~b}$ ).

\section{Discussion and conclusions}

The current best estimates of the beam width for the $850 \mathrm{GHz}$ horn indicate that the beam will be close to the design width. This estimate is provisional - the GRASP model uses the best videogrammetry-based estimates of the mirror shapes and the metrology of the telescope assembly to position the mirrors relative to each other, and the final pre-launch beam width estimate has been made taking the focal plane metrology into account in the model. Upcoming in-flight measurements of planets will enhance current knowledge.

The modelling of the propagation of the modes inside the optical chain is complex which required the development of novel experimental and theoretical techniques. There remain two major unknowns that will determine the beam width on the sky: (i) modal filtering in the flight model horn assembly, and (ii) the coupling of the detector in the cavity to the modes in the hornwaveguide structures. Mode filtering cannot be known until after launch, but damping is probably strong enough to prevent excessively narrow beams. Observation of planets with the multi-mode channels will yield the first accurate measurement of beam patterns.

The current knowledge base provides a good starting point for the in-flight optical calibration activities, which are expected to complement them. The current expectation is that, with the combination of ground knowledge and flight measurements, Planck will be able to achieve its main optical requirements.

\section{Acknowledgments}

The authors would like to acknowledge the support from STFC, CNRS, CNES, NASA, Enterprise Ireland and Science Foundation Ireland. The authors extend their gratitude to numerous engineers and scientists who have contributed to the design, development, construction or evaluation of HFI. 


\section{References}

[1] J. Tauber et al., Planck pre-launch status: the optical system of Planck, submitted to Astron. Astrophys. (2009).

[2] J. Tauber et al., Planck pre-launch status: the Planck mission, submitted to Astron. Astrophys. (2009).

[3] M. Bersanelli et al., Planck pre-launch status: design and description of the Low Frequency Instrument, accepted by Astron. Astrophys. (2009).

[4] J-M. Lamarre et al, Planck pre-launch status: the HFI instrument, submitted to Astron. Astrophys (2009).

[5] J.A. Murphy, R. Colgan, C. O’Sullivan, B. Maffei and P. Ade, Radiation patterns of multi-mode corrugated horns for far-IR space applications, Infrared Phys. Technol. 42 (2001) 515.

[6] R. Colgan, Electromagnetic and quasi-optical modelling of horn antennas for far-infrared space applications, Ph.D. thesis, NUI Maynooth (2001).

[7] E. Gleeson, Single and multi-mode corrugated horn design for cosmic microwave background experiments, Ph.D. thesis, NUI Maynooth (2004).

[8] F. Noviello, Optical performance of the ESA Planck surveyor and techniques for the study of the cosmic microwave background, Ph.D. thesis, NUI Maynooth (2008).

[9] J.J. Bock et al., A novel bolometer for infrared and millimeter-wave astrophysics, Space Sci. Rev. 74 (1995) 229.

[10] S. Withington and J.A. Murphy, Modal analysis of partially coherent submillimeter-wave quasi-optical systems, IEEE Trans. Antennas Propagat. 46 (1998) 1651.

[11] B. Maffei et al., Shaped corrugated horns for cosmic microwave background anisotropy measurements, Int. J. Infrared Milli. 21 (2000) 2023.

[12] P.A.R. Ade et al., Planck pre-launch status: the optical architecture of the HFI, submitted to Astron. Astrophys (2009).

[13] R.J.B. Clarricoats and A.D. Olver, Corrugated Horns for Microwave Antennas, IEE Electromagnetic Waves Series, Peter Peregrinus, London U.K. (1984).

[14] A.D. Olver, P.J.B. Clarricoats, A.A. Kishk and L Shafai, Microwave horns and feeds, IEEE Press, New York U.S.A. (1994).

[15] E. Gleeson et al., Corrugated waveguide band edge filters for CMB experiments in the far infrared, Infrared Phys. Technol. 46 (2005) 493.

[16] V.B. Yurchenko, J.A. Murphy and J-M. Lamarre, Fast physical optics simulations of the multi-beam dual-reflector submillimeter-wave telescope on the ESA PLANCK surveyor, Int. J. Infrared Milli. 22 (2001) 173.

[17] V.B. Yurchenko, J.A. Murphy, J-M. Lamarre and J. Brossard, Gaussian fitting parameters of the ESA Planck HFI beams, Int. J. Infrared Milli. 25 (2004) 601.

[18] J. Brossard, Couplage optique du télescope PLANCK avec l'instrument HFI: Modélisation et Caractérisation, Ph.D. thesis Université Paul Sabatier, Toulouse III (2001).

[19] B. Maffei et al., Planck pre-launch status: Planck-HFI optical optimization and beam performances, submitted to Astron. Astrophys. (2009). 
[20] C. del Rio Bocio et al., Optimal horn antenna design to excite high-order Gaussian beam modes from TEmO smooth circular waveguide modes, IEEE Trans. Antennas Propag. 47 (1999) 1440.

[21] J-M. Lamarre et al., The Planck High Frequency Instrument, a third generation CMB experiment, and a full sky submillimeter survey, New Astron. Rev. 47 (2003) 1017.

[22] C. O'Sullivan et al., The quasi-optical design of the QUaD telescope,Infrared Phys. Techn. 51 (2008) 277.

[23] J.A. Murphy et al., Millimeter-wave profiled corrugated horns for the QUaD cosmic background polarization experiment, Int. J. Infrared Milli. 26 (2005) 505.

[24] S. Church et al., Compact high-efficiency feed structure for cosmic microwave background astronomy at millimetre wavelengths, in Proceedings of the 30th ESLAB Symposium on Submillimetre and Far-Infrared Space Instrumentation, ESA-ESTEC (1996), pg. 77.

[25] D.H. Martin, Improved methods of cosmic microwave background radiometry, in Gamov Cosmology 86, F. Melchiori and R. Ruffini eds., North Holland, Amsterdam (1986).

[26] E. Gleeson, J.A. Murphy and B. Maffei, "Phase centers" of far infrared multi-mode horn antennas, Int. J. Infrared Milli. 23 (2002) 711.

[27] A. Benoit et al., Archeops: a high resolution, large sky coverage balloon experiment for mapping cosmic microwave background anisotropies, Astropart. Phys. 17 (2002) 101.

[28] G.M. Smith et al., Removing standing waves in quasi-optical systems by optimal feedhorn design, in IRMMW-THz 2007, pg. 941.

[29] P.A.R. Ade and G. Savini, in preparation for submission to JINST (2010). 